\title{
Retrospective Analysis of Combined Treatment with Triple Combination Cream and Hyaluronic Acid Microneedle Patch for Benign Pigmented Skin Lesions
}

\author{
Je Hyuk Lee ${ }^{1}$, Min Seung Kim², Jong Woo Kim³ ${ }^{3}$ Wook $\mathrm{Oh}^{4}$, Hyoung Moon Kim ${ }^{5}$ and Kee Hwan \\ Kwon ${ }^{6 *}$ \\ ${ }^{1}$ Doctorbom Clinic, Seoul, Republic of Korea \\ ${ }^{2}$ INEE Clinic, Seoul, Republic of Korea
}

${ }^{3} I U$ Clinic, Seoul, Republic of Korea

${ }^{4}$ Samsung Feel Clinic, Seoul, Republic of Korea

${ }^{5}$ Maylin Clinic, Gyeonggi-do, Republic of Korea

${ }^{6}$ Department of Otolaryngology Head and Neck Surgery, Kangdong Sacred Heart Hospital, Hallym University College of Medicine, 150, Republic of Korea

*Corresponding author: Kee Hwan Kwon, Department of Otolaryngology Head and Neck Surgery, Kangdong Sacred Heart Hospital, Hallym University College of Medicine, Republic of Korea

\section{ARTICLE INFO}

Received: 崫 December 16, 2020

Published: 幽 January 19, 2021

Citation: Je Hyuk Lee, Min Seung Kim, Jong Woo Kim, Wook Oh, Hyoung Moon Kim, Kee Hwan Kwon. Retrospective Analysis of Combined Treatment with Triple Combination Cream and Hyaluronic Acid Microneedle Patch for Benign Pigmented Skin Lesions. Biomed J Sci \& Tech Res 33(2)-2021. BJSTR. MS.ID.005372.

Abbreviations: ANOVA: Analysis of Variance; UV: Ultra-Violet; TCC: Triple Combination Cream; HA: Hyaluronic Acid

\begin{abstract}
Background: Triple Combination Cream (TCC), which is a combination of retinoic acid, hydroquinone, and a corticosteroid at fixed ratios, is an economical option for treating common pigmented skin lesions, although it can elicit some discomfort, including redness, dryness, and itching.
\end{abstract}

Objective: We evaluated the clinical usefulness of combining TCC with a dissolvable microneedle hyaluronic acid patch (TCC+HA-DMN) to achieve the therapeutic effects of TCC while avoiding its side effects when treating pigmented skin lesions.

Methods \& Materials: We retrospectively analyzed the results of 25 patients (mean age, 48.2 years, range $27-72$ years, $S D=13.078$ ) with pigmented lesions along the face (e.g., solar lentigines and seborrheic keratoses) who received TCC+HA-DMN between March 1, 2018 and June 30, 2019. For 4 weeks, patients were asked to apply TCC and an HA-DMN patch daily only to the pigmented area and to wash their face after 3 hours.

Results: A UV and polarized LED light source and CMOS (Complementary metal-oxidesemiconductor) sensor integrated digital analysis system revealed significant reductions in pigmentation and melanin level in lesions 4 and 8 weeks after initiating TCC+HA-DMN, respectively. Visual analog scale scores also highlighted marked improvements in lesion appearance at 4 and 8 weeks $(\min =1, \max =5$, mean $=2.48$ (4weeks), 3.76 ( 8 weeks); standard deviation at 4 and 8 weeks $=0.871$ and 1.051, respectively).

Conclusion: TCC+HA-DMN is effective in treating pigmented skin lesions.

\section{Introduction}

Benign pigmented skin lesions, particularly solar lentigines and seborrheic keratoses, commonly spur visits to a dermatologist. Lentigines are spots of melanin pigment between keratinocytes and melanocytes in the epidermis and appear upon exposure to Ultra-Violet (UV) rays. Seborrheic keratoses are similar to solar lentigines but appear as thick keratin lesions in the skin. Research 
has indicated that solar lentigines show structural changes in the epidermis that are accompanied by increases in the expression of genetic markers associated with melanin synthesis, such as proopiomelanocortin, tyrosinase, tyrosinase-related protein 1, dopachrome tautomerase, Pmel-17, and microphthalmiaassociated transcription factor [1]. These changes in the skin are permanent and do not recover by avoiding exposure to UV light. Thus, clinical intervention is necessary to remove solar lentigines.

Solar lentigine treatment can be broadly divided into two categories: physical removal and topical applications. Topical therapy is relatively cost-effective and primarily relies on the use of triple combination cream (TCC), which is a combination of retinoic acid, hydroquinone, and corticosteroid at fixed ratios [2]. Hydroquinone suppresses tyrosinase and reduces the formation of melanin pigment; retinoic acid promotes keratinocyte loss and the downregulation of tyrosinase; and the steroid suppresses tyrosinase gene transcription, thus providing an anti-inflammatory effect. Altogether, they provide a skin lightening effect [2]. In a review of clinical studies conducted over the past 20 years, the Pigmentary Disorders Academy has recommended TCC as the first option for treating patients with pigmented blemishes [3].

The typical drawbacks of topical TCC treatment include irritant dermatitis with keratin, dryness, and erythema caused by retinoic acid [4]. An irritant to the skin, retinoic acid has low solubility in water, is unstable in light, and has low permeability in the skin. Interestingly, in a study of lentigo and seborrheic keratosis treatment, [5] noted an improvement in treatment results when using a microneedle patch loaded with retinoic acid to overcome the drawbacks of monotherapy with retinoic acid. In another study, [6] indicated that the use of hydroquinone ointment and silicone sheets containing retinoic acid to treat epidermal melanosis elicited less skin irritation and had equivalent treatment effects, compared to when using hydroquinone ointment alone. Thus, TCC treatment for pigmented lesions may also benefit from the application of similar transdermal drug delivery systems. Accordingly, in this study, we sought to evaluate the effectiveness of combined treatment of TCC and a dissolvable microneedle patch loaded with hyaluronic acid (TCC + HA-DMN patch) for localized, benign, pigmented skin lesions. Through a retrospective review of medical records and results from digital analyses of the skin before and at 4 and 8 weeks after initiating treatment, we assessed potential reductions in both pigmentation and the common side effects of TCC (e.g., redness, dryness, and itching).

\section{Methods}

\section{Study Design and Subjects}

This retrospective study was approved by the Public Institutional Bioethics Committee, which is designated by the Ministry of Health and Welfare in Seoul, Korea on October 28, 2019 (P01-201910-21-016). Among all patients who visited one of five research institutes from March 12018 to June 302019 and who were treated with a TCC + HA-DMN patch for local pigmented lesions on the face, we extracted and reviewed the medical records and images of 25 patients who met the following selection criteria:

a. Diagnosed with local solar lentigines, seborrheic keratoses, or freckles;

b. Age older than 20 years;

c. Received TCC + an HA-DMN patch instead of laser or surgical treatment; and

d. Available imaging data at baseline before treatment and at 4 and 8 weeks after initiating treatment.

Patients were excluded if they met any of the following criteria:

a. Were undergoing systemic or local immunotherapy for atopic dermatitis, psoriasis, or lupus erythematosus, among others;

b. Had seborrheic dermatitis;

c. Had lichen simplex chronicus;

d. Had malignant or premalignant skin disease;

e. Had an infectious disease; or

f. Other features deemed unsuitable for study participation based on the clinical judgment of the authors.

\section{Study Materials}

TCC (Trilustra ${ }^{\circledR}$ ) was purchased from Kolmar Korea (Seoul, South Korea) and contained $0.5 \mathrm{mg} / \mathrm{g}$ of tretinoin, $40 \mathrm{mg} / \mathrm{g}$ of hydroquinone, and $0.1 \mathrm{mg} / \mathrm{g}$ of fluocinolone acetonide. The HADMN patches (Thera-Pass $₫$ RMD-6.5B) were purchased from the RAPHAS Corporation (Cheon An, South Korea). The dissolvable microneedles on each patch contained $100 \%$ hyaluronic acid and comprised a needle length of $650 \mu \mathrm{m}$. For digital pigmentation analysis, we used the Mark-Vu® skin analyzing system (PSI Plus, Suwon, South Korea). The Mark-Vu® system has four different LED-type light sources (normal, specular, polarized, UV), a CMOS (Complementary metal-oxide-semiconductor) sensor of 18.0 mega pixels, and integrated analysis tools (Figure 1). Epidermal pigment and melanin level analyses use numerical data to represent the amount of melanin and degree of pigmentation.

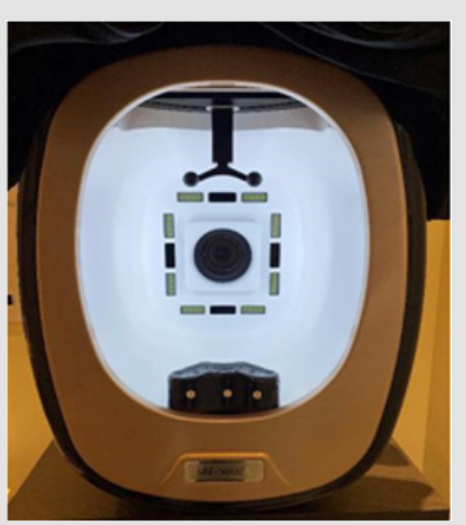

Figure 1: Mark-Vu® Skin Analysis system. 


\section{Procedures}

Instead of the conventional method of applying TCC at night before bedtime and washing it off the next day, we asked all 25 patients to apply the cream only to the pigmented area and to then put the HA-DMN patch on top, as described in Figure 2. After 3 hours, they were to then wash their face. Patients were to apply the combined treatment only on solar lentigines lesions, even if other melasma or acquired bilateral nevus of Ota-like macule lesions were present. The treatment was to be applied once daily for up to 4 weeks.

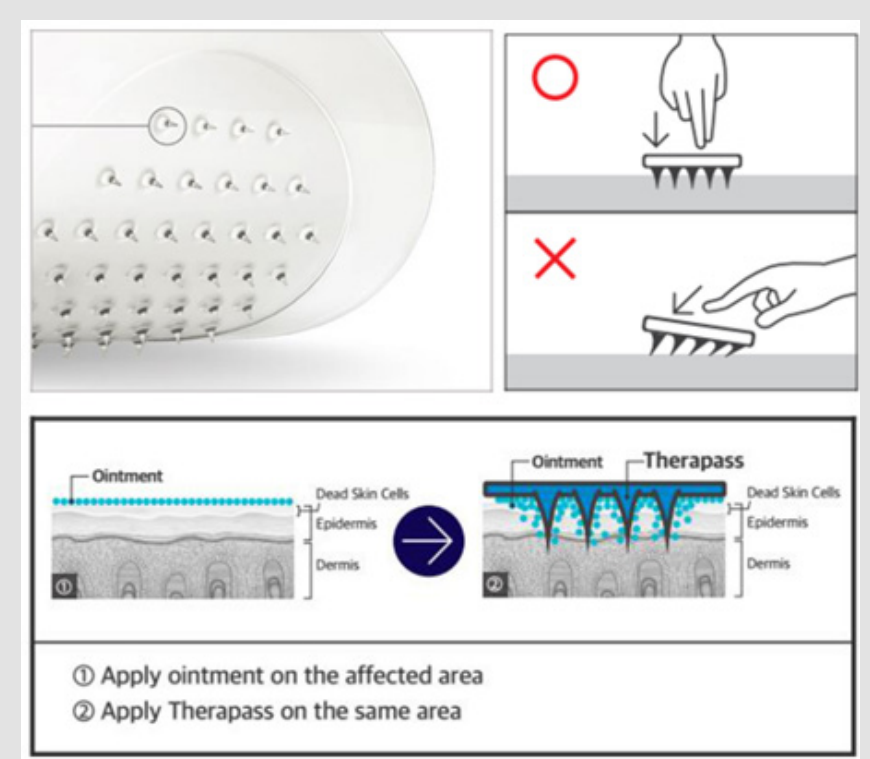

Figure 2: Image of a Thera-Pass ${ }^{\circledR}$ RMD-6.5A microneedle patch and an illustration of how the patch ought to be applied to the skin. Image is the courtesy of the RAPHAS Corporation.

\section{Clinical Assessment}

Melanin levels and epidermal pigmentation were objectively assessed using a digital facial analyzer (Mark-Vuß) before treatment, after 4 weeks of treatment, and after an additional 4 weeks of follow up. The Mark-Vu® analyzer utilizes focused area analysis to optically quantify the degree of pigmentation and melanin amount of specific areas (Figure 3) and show a numerical value along the areas where the TCC + HA-DMN treatment was applied. Data were recorded and analyzed pre- and posttreatment. Measurement of skin color is an accepted method of assessing melanin content and pigmentation levels. Additionally, digital images of the targeted area under natural and polarized, UV light were also taken at the same points in time (Figures 4 \& 5). The degree of improvement in pigmentation of the targeted lesions was subjectively evaluated as 1 (worse), 2 (no change), 3 (<30\% improvement), 4 (30-60\% improvement), and 5 ( $>60 \%$ improvement), by a treating physician using a visual analogue scale (VAS) 4 and 8 weeks after initiating treatment (Figure 6). Further, the presence or absence of any treatment-related discomfort was recorded, describing the frequency, type, and number of instances.

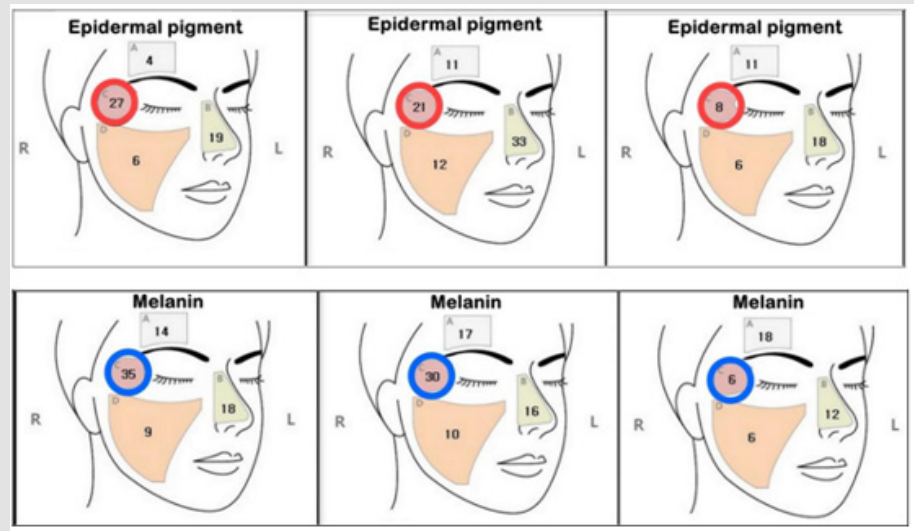

Figure 3: Representative example of changes in the numerical values given by Mark-Vu® in the C region (left: before treatment, center: 4 weeks after initiating treatment, right: 8 weeks after initiating treatment). 


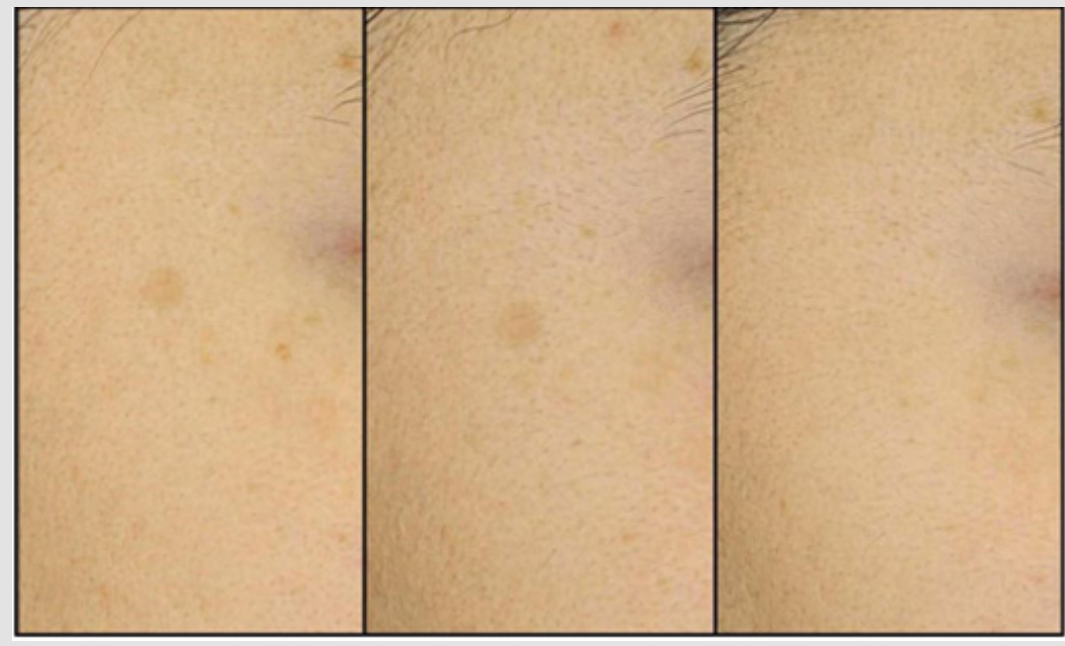

Figure 4: Digital image of pigmentation in patient A under natural light (left: before, center: 4 weeks, right: 8 weeks).

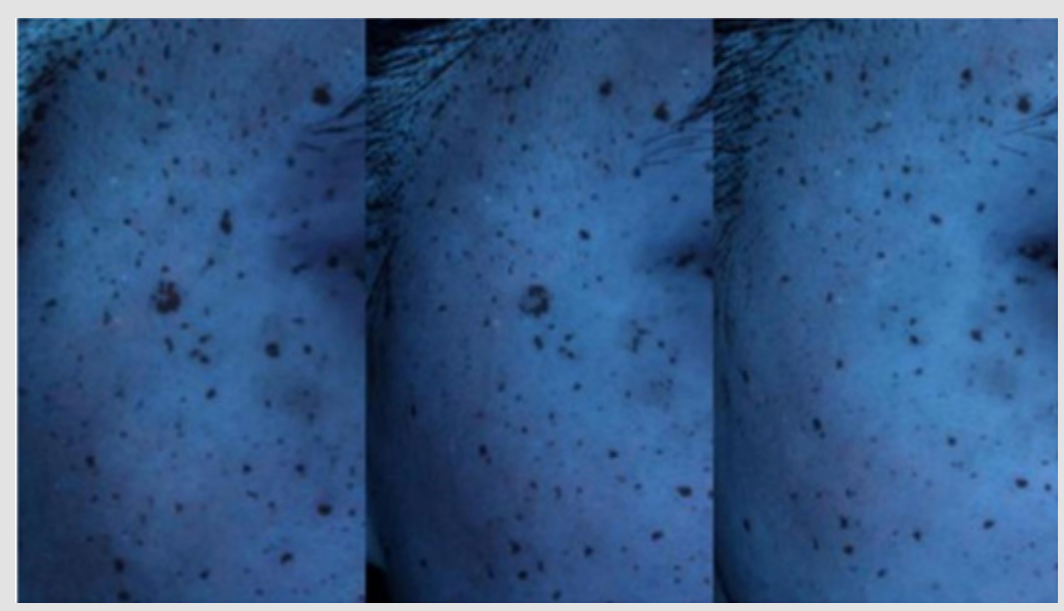

Figure 5: Digital image of pigmentation in patient A under ultra-violet light (left: before, center: 4 weeks, right: 8 weeks).

\section{Subjective clinical assessment was evaluated on a visual analog scale (VAS) as 1 (worse),} 2 ( no change), $3(<30 \%), 4(30-60 \%)$, and $5(>60 \%)$ by a treating physician

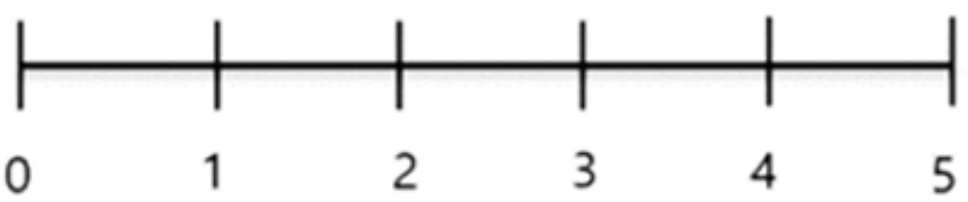

Figure 6: Visual analogue scale for the subjective assessment of improvements in pigmented lesions.

\section{Statistical Analysis}

A repeated measures analysis of variance (ANOVA) and nonparametric verification with the Friedman test, along with a post-hoc Bonferroni correction, were conducted to evaluate the significance of changes in epidermal pigmentation and melanin over time. The Shapiro-Wilk and Kolmogorov-Smirnov tests were performed to test for normality. All statistical analyses were conducted using SPSS software, version 26.0 (SPSS, Inc., Chicago, IL, USA).

\section{Results}

Patient characteristics and their diagnoses are listed in Table 1. Among the 25 cases selected for analysis, 24 were women, and 
the average age was 48.2 years. Overall, 92\% (n=23) and 96\% $(n=24)$ of the patients experienced objective improvements in epidermal pigmentation and melanin level, respectively, at 8 weeks after initiating treatment (Figure 7). The average numerical value generated using the Mark-Vuß analyzer for epidermal pigmentation revealed an improvement of $20.46 \%$ after 8 weeks, dropping from 32.64 before 3 treatment to 25.96 ( $p=0.002$ ) (Figure 8 \& Table 2). Melanin improved by $16.6 \%$, decreasing from 47.92 before treatment to 29.96 after 8 weeks ( $p<0.001$ ). The average VAS scores for subjective improvement in the appearance of the target lesions compared to those before treatment were 2.48 at 4 weeks $(p=0.871)$ and 3.76 at 8 weeks $(p<0.001)$, indicating greater improvement at an additional 4 weeks after treatment. The repeated measures ANOVA and non-parametric verification with the Friedman test indicated that the changes in the numerical values for epidermal pigmentation and melanin over time were significant (Table 2). The changes in epidermal pigmentation had an F value of 11.838 and a significance probability of 0.002 (Table 3). The changes in melanin had an F value of 32.837 and a significance probability $<0.001$. In total, 10 patients reported experiencing side effects with TCC + HA-DMN, and 4 patients experienced two or more side effects, including itching $(\mathrm{n}=1)$, erythema $(\mathrm{n}=8)$, dryness $(n=2)$, photosensitivity $(n=1)$, and pigmentation $(n=4)$ (Table 4). Generally, symptoms gradually subsided with continued use and naturally disappeared approximately 1 week after treatment was completed.'

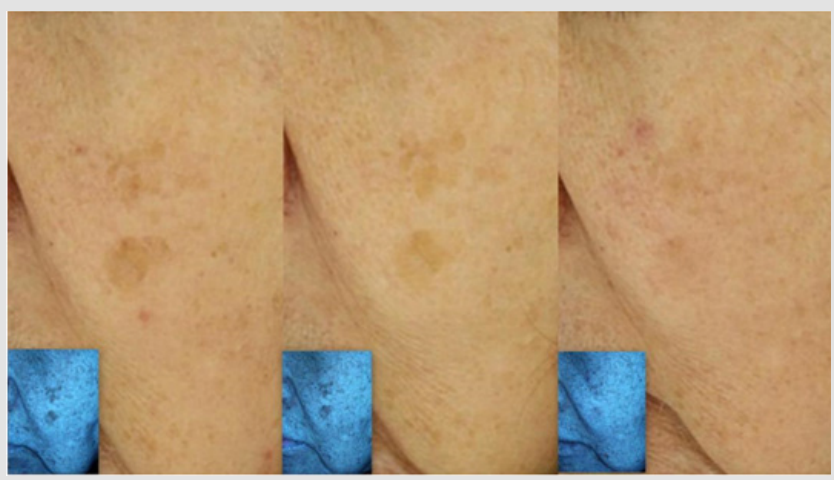

Figure 7: Representative image of improvement in pigmentation 4 and 8 weeks after initiating combined treatment with triple combination cream and a dissolvable microneedle patch of hyaluronic acid.

Table 1: Patient characteristics.

\begin{tabular}{|c|c|}
\hline Patients & $\mathbf{n = 2 5}$ \\
\hline Sex & \\
\hline Male & $1(4 \%)$ \\
\hline Female & $24(96 \%)$ \\
\hline Age & $27 \sim 72$ \\
\hline Mean/SD & $48.16 / 13.078$ \\
\hline Clinical diagnosis & \\
\hline Lentigo & $25(100 \%)$ \\
\hline
\end{tabular}

\begin{tabular}{|c|c|}
\hline PIH & $1(4 \%)$ \\
\hline ABNOM & $1(4 \%)$ \\
\hline Melasma & $3(12 \%)$ \\
\hline SK & $1(4 \%)$ \\
\hline
\end{tabular}

Note: PIH, Post inflammatory hyperpigmentation; ABNOM, Acquired bilateral nevus of Ota like macules; SK, Seborrheic keratosis.

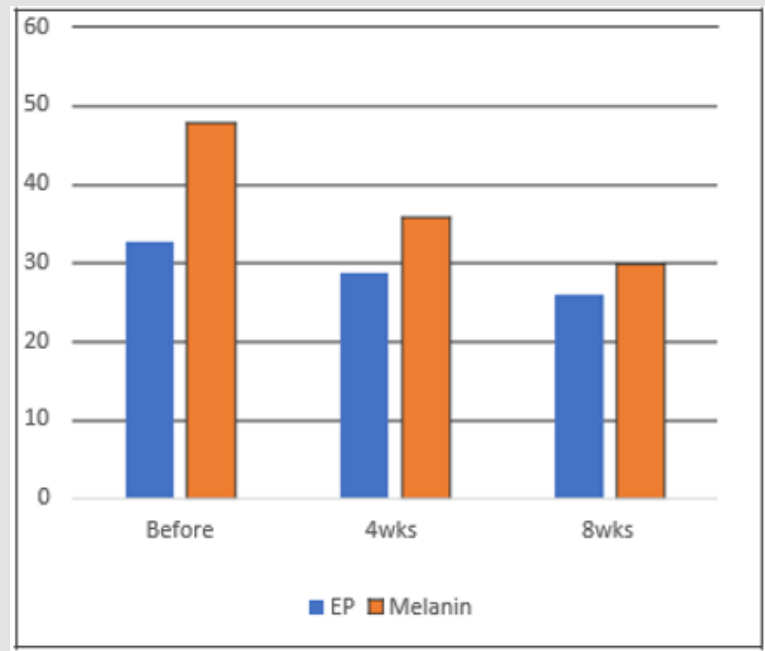

Figure 8: Bar graph depicting changes in epidermal pigmentation (EP) and melanin at 4 and 8 weeks after initiating combined treatment with triple combination cream and a dissolvable microneedle patch of hyaluronic acid.

Table 2: Changes in epidermal pigmentation and melanin over time.

\begin{tabular}{|c|c|c|c|c|c|c|}
\hline \multicolumn{7}{|c|}{ Repeated ANOVA - Bonferroni Test } \\
\hline & & Mean & SD & F & P & $\begin{array}{c}\text { Post-Hoc } \\
\text { test }\end{array}$ \\
\hline & Before & 32.64 & 8.93 & & & \\
\hline EP & $4 w k s$ & 28.68 & 9.424 & & & \\
\hline & $8 w k s$ & 25.96 & 12.064 & 11.838 & $0.002^{* *}$ & $\mathrm{a}>\mathrm{b}, \mathrm{c}$ \\
\hline & Before(a) & 47.92 & 16.462 & & & \\
\hline Melanin & 4 wks(b) & 35.84 & 16.486 & & & \\
\hline & $8 w k s(c)$ & 29.96 & 16.177 & 32.87 & $.000^{* *}$ & $\mathrm{a}>\mathrm{b}, \mathrm{c}$ \\
\hline
\end{tabular}

Note: Repeated ANOVA and Friedman test results, with a Bonferroni correction.

* $\mathrm{p}<0.05$, ** $\mathrm{p}<0.01$; EP, epidermal pigmentation; ANOVA, analysis of variance.

Table 3: Shapiro-Wilk and Kolmogorov-Smirnov test results for normality.

\begin{tabular}{|c|c|c|c|c|}
\hline & \multicolumn{2}{|c|}{ Kolmogorov-Smirnov } & \multicolumn{2}{c|}{ Shapiro-Wilk } \\
\hline Statistic & $\begin{array}{c}\text { CTT } \\
\text { Significance } \\
\text { probability (p) }\end{array}$ & Statistic & $\begin{array}{c}\text { CTT } \\
\text { Significance } \\
\text { probability (p) }\end{array}$ \\
\hline EP Before & 0.167 & 0.069 & 0.951 & 0.259 \\
\hline EP 4wks & 0.108 & $0.200^{*}$ & 0.97 & 0.634 \\
\hline
\end{tabular}




\begin{tabular}{|c|c|c|c|c|}
\hline EP 8wks & 0.12 & $0.200^{*}$ & 0.956 & 0.338 \\
\hline $\begin{array}{c}\text { Melanin } \\
\text { Before }\end{array}$ & 0.114 & $0.200^{*}$ & 0.953 & 0.288 \\
\hline $\begin{array}{c}\text { Melanin } 4 \\
\text { wks }\end{array}$ & 0.24 & 0.001 & 0.916 & 0.042 \\
\hline $\begin{array}{c}\text { Melanin 8 } \\
\text { wks }\end{array}$ & 0.146 & 0.175 & 0.934 & 0.105 \\
\hline $\begin{array}{c}\text { VAS 4 } \\
\text { wks }\end{array}$ & 0.229 & 0.002 & 0.882 & 0.008 \\
\hline Vas 8 wks & 0.27 & 0 & 0.868 & 0.004 \\
\hline
\end{tabular}

Note: * $\mathrm{p}>0.05$, Normality distribution; CTT, classical test theory; EP, epidermal pigmentation; VAS, Visual analog scale.

Table 4: Side effects of combined treatment with triple combination cream and a dissolvable microneedle patch of hyaluronic acid.

\begin{tabular}{|c|c|c|}
\hline Side Effects & + /- & $\mathbf{n}(\%)$ \\
\hline \multirow{2}{*}{ Itching } & + & $1(4)$ \\
\hline \multirow{2}{*}{ Erythema } & - & $24(96)$ \\
\hline Dryness & + & $8(32)$ \\
& - & $17(68)$ \\
\hline \multirow{2}{*}{ Photosensitivity } & + & $2(8)$ \\
& + & $23(92)$ \\
\hline \multirow{2}{*}{ Pigmentation } & + & $1(4)$ \\
& - & $24(96)$ \\
\hline
\end{tabular}

Note: All symptoms resolved naturally within 1 week after the treatment was discontinued.

\section{Discussion}

In this study, we aimed to determine whether adding an HADMN patch to TCC would be beneficial for simple treatment of benign pigmented lesions in the skin. Indeed, we found that the combined treatment not only was effective at treating such lesions, facilitating significant reductions in both epidermal pigmentation and melanin content, but also elicited discomfort to a lesser or similar degree as that for comparable methods. Topical application of TCC is a simple and economical option for treating pigmented lesions of the skin. The advantages of laser treatment for solar lentigines include the potential for permanent removal in as little as one treatment session depending on the lesion, a constant increase in the therapeutic effect with a greater number of treatments, a short treatment duration, and a relatively longer lasting therapeutic effect.

Some common lasers that are used at this time include the 532 $\mathrm{nm}$ frequency-doubled Q-Switched (QS), QS-ruby, QS-alexandrite, long-pulsed potassium-titanyl-phosphate, long-pulsed alexandrite, and erbium lasers $[2,7,8]$. In a 12 -week follow-up study which compared topical TCC treatment to QS-ruby laser treatment for solar lentigines, both patients and physicians found the QS-ruby

laser to be superior in terms of how quickly a therapeutic effect was achieved, treatment duration, and degree of improvement [9]. However, laser treatment does pose disadvantages, including a relatively high treatment cost and a reduced therapeutic effect depending on the degree of pigmentation or the concentration of chromophores in the lesions. Moreover, particularly in the case of $532 \mathrm{~nm}$ laser treatment, possible side effects such as postinflammatory hyperpigmentation, hypopigmentation, and uneven pigmentation can occur in patients with darker skin tones or with repeated treatment of recurrent lesions. Further, with ablative lasers, side effects such as local tissue depression and burns may occur $[7,8,10]$. Accordingly, in light of the present results, we suggest that the use of combined TCC and HA-DMN patch treatment, as described in this study, may be beneficial for patients who are prone to adverse effects with laser treatment, particularly Asian patients who show a high incidence of post-inflammatory hyperpigmentation [5].

Among several transdermal drug delivery systems, microneedle patches tend to facilitate drug delivery to the skin better than sheets. Microneedles generally comprise a hollow or solid needle structure that does not exceed an approximate length of 50-900 $\mu \mathrm{m}$ and a thickness of $300 \mu \mathrm{m}$. They also offer advantages, including the painless administration of large molecular substances and fast healing at the injection site. Moreover, they elicit no fear among patients who are afraid of needles and can be used to selectively deliver drugs to specific areas of the skin.13 Microneedles can be solid or dissolvable; dissolvable microneedles are made of polymers or water-solvable carbohydrates that naturally decompose when inserted into the skin, such as maltose, trehalose, polyvinyl alcoholcarboxymethyl cellulose, silk, gelatin, and hyaluronic acid [11]. In a study of an HA-DMN patch loaded with retinoic acid for the treatment of seborrheic keratosis and senile lentigo, Hirobe et al. 9 explained that the penetrating hyaluronic acid microneedles weakened the stratum corneum by inducing edema, allowing retinoic acid to permeate the epidermis.

As an added benefit, the hyaluronic acid component helped to moisturize the epidermis as the microneedles dissolved 9; when moisture levels increase and percutaneous moisture loss decreases, dryness, wrinkles, and pruritus generally improve. In another study, the HA-DMN patch alone was found to improve the appearance of eye wrinkles, [12] while further research indicated that the use of a combination of adenosine wrinkle cream and an HA-DMN patch elicited superior treatment results, above and beyond using each treatment separately, without any notable side effects [13]. Additionally, an in vivo study of a microneedle patch in humans demonstrated that local skin side effects, such as erythema, mostly disappeared within 7 days after patch application and that skin returned to normal within 30 days [14-16]. This study has a few limitations that warrant consideration. First, due to the retrospective nature of this study and the small number of patients, we were unable to draw definitive conclusions regarding the 
efficacy of the combined treatment presented herein [17]. Finally, there may be some recall bias in the reporting of side effects by the patients [18].

\section{Conclusion}

In light of the present results, we suggest that combined TCC and HA-DMN patch treatment, as described in this study, which shortens the length of time that TCC is applied to the skin (3 hours vs. overnight), may be beneficial for treating benign pigmented skin lesions in patients who are prone to discomfort when they apply cream and to adverse effects of laser treatment. The intermittent application of TCC in combination with an HA-DMN patch showed therapeutic effects equivalent to conventional all-day application of TCC, with fewer side effects, and the effects of the combined treatment were more pronounced 4 weeks after treatment was completed. Further research is needed to devise an optimal treatment regimen for combining TCC with an HA-DMN patch.

\section{Acknowledgement}

I wish to thank my fellow Seung Min Oh for his assistance in this article.

\section{Conflicts of Interest}

The authors declare no potential conflicts of interest.

\section{References}

1. Ortonne JP, Pandya AG, Lui H, Hexsel D (2006) Treatment of solar lentigines. J Am Acad Dermatol 54: S262-S271.

2. Ortonne JP, Bissett DL (2008) Latest insights into skin hyperpigmentation. J Investig Dermatol Symp Proc 13(1): 10-14.

3. Al-Dujaili Z, Dierickx CC (2013) Laser treatment of pigmented lesions. In: Goldberg DJ, editor. Laser dermatology. Berlin, Heidelberg: Springer Berlin Heidelberg p. 41-64.

4. Bukvic Mokos Z, Lipozencic J, Pasic A, Fattorini I (2006) Laser therapy for solar lentigines: review of the literature and case report. Acta Dermatovenerol Croat 14(2): 81-85.

5. Hirobe S, Otsuka R, Iioka H, Quan YS, Kamiyama F, et al. (2017) Clinical study of a retinoic acid-loaded microneedle patch for seborrheic keratosis or senile lentigo. Life Sci 168: 24-27.

6. Iida S, Takushima A, Ohura N, Sato S, Kurita M, et al. (2013) Silicone sheet containing all-trans retinoic acid and hydroquinone for the treatment of epidermal melanosis. Dermatol Surg 39(8): 1237-1242.

\section{ISSN: 2574-1241}

DOI: 10.26717/BJSTR.2021.33.005372

Kee Hwan Kwon. Biomed J Sci \& Tech Res

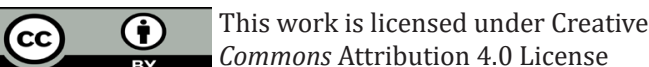

Submission Link: https://biomedres.us/submit-manuscript.php
7. Chan HHL (2011) Pigmentation and hypopigmentation: benign pigmented lesions. In: Raulin C, Karsai S, editors. Laser and IPL technology in dermatology and aesthetic medicine. Springer Berlin Heidelberg, Heidelberg, Berlin, Germany, pp. 147-155.

8. Imhof L, Dummer R, Dreier J, Kolm I, Barysch MJ (2016) A prospective trial comparing Q-switched ruby laser and a triple combination skinlightening cream in the treatment of solar lentigines. Dermatol Surg 42(7): 853-857.

9. Chan R, Park KC, Lee MH, Lee ES (2008) A randomized controlled trial of the efficacy and safety of a fixed triple combination (fluocinolone acetonide $0.01 \%$, hydroquinone $4 \%$, tretinoin $0.05 \%$ ) compared with hydroquinone $4 \%$ cream in Asian patients with moderate to severe melasma. Br J Dermatol 159(3): 697-703.

10. Draelos ZD (2007) Skin lightening preparations and the hydroquinone controversy. Dermatol Ther 20(5): 308-313.

11.Wang QL, Zhang XP, Chen BZ, Guo XD (2018) Dissolvable layered microneedles with core-shell structures for transdermal drug delivery. Mater Sci Eng C Mater Biol Appl 83: 143-147.

12. Choi SY, Kwon HJ, Ahn GR, Ko EJ, Yoo KH, et al. (2017) Hyaluronic acid microneedle patch for the improvement of crow's feet wrinkles. Dermatol Ther 30(6): e12546.

13. Hong JY, Ko EJ, Choi SY, Li K, Kim AR, et al. (2018) Efficacy and safety of a novel, soluble microneedle patch for the improvement of facial wrinkle. J Cosmet Dermatol 17(2): 235-241.

14. Hiraishi Y, Hirobe S, Iioka H, Quan YS, Kamiyama F, et al. (2013) Development of a novel therapeutic approach using a retinoic acidloaded microneedle patch for seborrheic keratosis treatment and safety study in humans. J Control Release 171(2): 93-103.

15. Liu S, Jin MN, Quan YS, Kamiyama F, Katsumi H, et al. (2012) The development and characteristics of novel microneedle arrays fabricated from hyaluronic acid, and their application in the transdermal delivery of insulin. J Control Release 161(3): 933-941.

16. Ji J, Tay FEH, Miao J, Iliescu C (2006) Microfabricated microneedle with porous tip for drug delivery. J Micromech Microeng 16(5): 958-964.

17. Bariya SH, Gohel MC, Mehta TA, Sharma OP (2012) Microneedles: an emerging transdermal drug delivery system. J Pharm Pharmacol 64(1): 11-29.

18. Nasrollahi SA, Nematzadeh MS, Samadi A, Ayatollahi A, Yadangi S, et al. (2019) Evaluation of the safety and efficacy of a triple combination cream (hydroquinone, tretinoin, and fluocinolone) for treatment of melasma in Middle Eastern skin. Clin Cosmet Investig Dermatol 12: 437444 . 